
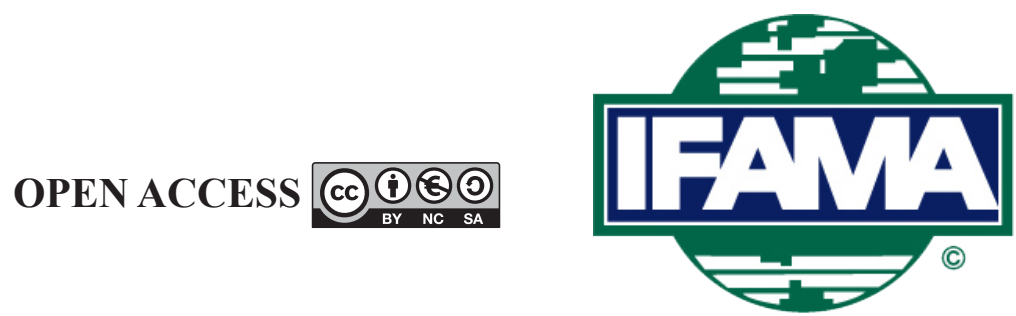

International Food and Agribusiness Management Review

Volume 23, Issue 5, 2020; DOI: 10.22434/IFAMR2019.0124

Received: 31 July 2019 / Accepted: 22 October 2019

Special issue: New and sustainable food and agribusiness management models

\title{
Support policy preferences of grain family farms: evidence from Huang-huai-hai plain of China
}

\section{RESEARCH ARTICLE}

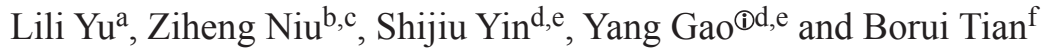

${ }^{a}$ PhD candidate, Graduate School of Economics, Ryukoku University, 67

Tsukamoto-cho, Fukakusa, Fushimi-ku, Kyoto, Japan

${ }^{b} \mathrm{MSc}$ candidate, ${ }^{\mathrm{d}}$ Professor, ${ }^{\mathrm{f}}$ Undergraduate student, College of Economics, Qufu

Normal University, Yantai Road 80\#, Rizhao, Shandong, China P.R.

${ }^{c}$ MSc candidate, ${ }^{e}$ Professor, Research Center for Food Safety and Agricultural Green

Development, Qufu Normal University, Yantai Road 80\#, Rizhao, Shandong, China P.R.

\begin{abstract}
This study uses the choice experiment method with 570 grain family farms located in the Huang-huai-hai Plain and determine various support policy attributes and the attribute levels for the two dimensions of policy measures and policy communication channels. Ordering effects are eliminated by warming up subjects in advance and using information disclosure. This paper uses the inferred attribute non-attendance method to process attributes ignored by the grain family farms and analyzes grain family farms' preferences for different support policies with a mixed logit model and then uses a latent class model to analyze how the characteristics of grain family farms relate to different preference types. We find that grain family farms have a strong preference for agricultural subsidies, credit support, and technical support (the mean coefficient is greater than 0.8). Moreover, the preferences of grain family farms over the policy communication channel (the mean coefficient is greater than 0.5 ) cannot be ignored. Faced with the same policy attribute combination, grain family farms with high education levels, reasonable scales of operation, and good understanding of support policies are more likely to improve their profit margins. There are four preference types of grain family farms: finance preference (43.2\%), knowledge and technology preference (28.5\%), land transfer preference $(15.4 \%)$, and policy information preference (12.9\%).
\end{abstract}

Keywords: grain family farms, attribute non-attendance, policy choice, mixed logit model, latent class model JEL code: Q12, Q18

${ }^{\circledR}$ Corresponding author: koyo718@163.com 


\section{Introduction}

Because family farms can ensure food security, reduce the income gap between urban and rural areas, and protect the environment (Gao et al., 2019a), China's 'No. 1 Central Document' has mentioned the government's goal 'to support the development of family farms' every year since 2013.

Foreign scholars have created relatively complete and effective theoretical and practical research systems for studying family farm support policies. Tzouramani et al. (2014) pointed out that the government should provide a stable financing environment and provide corresponding agricultural subsidies to increase the operating performance of organic cherry farms in northern Greece. McFadden and Gorman (2016) argued that the government should provide educational training and technical support to enhance the innovation capacity of Irish family farms. Medina et al. (2015) argued that targeted support for family farms in terms of capital, technology, and market resource integration can fully stimulate farms' developmental potential. Toader and Roman (2015) proved that investing in agricultural infrastructure, improving the agricultural services and carrying out agricultural technical training can promote the sustainable development of family farms.

Based on these and similar studies, governments have introduced many policies to support family farms (Vliet et al., 2015). The Common Agricultural Policy strongly influences the development of family farms in EU countries and provides a reference for other countries' policies for supporting family farms (Barnes et al., 2016). In the United States and Japan, family farm support policies mainly involve providing technology support, improving land systems, and optimizing the legal environment and agricultural services' systems (Hoppe and Banker, 2010; Kihl, 1982). Australia promotes family farm development through financing support, tax incentives, and aid committees (Botterill, 2016). The agricultural management pattern dominated by family farms vigorously supported by the government observed in agriculturally developed countries is a model for the successful operation of family farms (Wiggins et al., 2010).

Graeub et al. (2015) stressed that countries must craft their policies for family farms based on their country's particular conditions. There are great differences between China's growth stage, land system, agricultural subsidy system, agricultural services, and operator quality and those of developed countries (Gao et al., 2017a). Therefore, the applicability of the research results of foreign scholars and of the support policies introduced by foreign governments must be examined in the context of China.

Using studies of foreign countries and the situation of China's family farms as their basis, Chinese scholars have conducted extensive research into local policies for supporting family farms. Du and Xiao (2014) argued that when considering family farm support policies, the rural land system is the basic system environment, policy support forms a strong backbone, agricultural cultivation and training systems are important propellers of development, and agricultural services play an important role. Chinese scholars argue that the government should promote the orderly evolution of land norms (Zhu et al., 2016), increase the intensity of policy support (Yu and Chen, 2016), establish education and training systems (Gao et al., 2017a), and improve agricultural services (Lv and Zhu, 2015). Drawing on these studies, the Chinese government has introduced a series of policies for supporting family farms, including financial subsidies, credit support, agricultural insurance, education, training, and technical support ${ }^{1}$.

Thanks to the efforts of the central and local governments, China has more than 870,000 family farms covering an area of 176 million $\mathrm{mu}^{2}$ and accounting for $13.4 \%$ of total cultivated land (Gao et al., 2017b). However, the number of Chinese family farms is small compared with the more than 100 million traditional farmers ${ }^{3}$. The diffculties currently faced by family farms are inefficiencies in scale, barriers to credit financing

\footnotetext{
${ }^{1}$ China's 'No. 1 Central Document' of 2016 and 2017. http://www.moa.gov.cn/ztzl/2016zyyhwj/2016zyyhwj (2016) and http://www.moa.gov.cn/ ztzl/yhwj2017/zywj (2017). in Chinese.

$21 \mathrm{mu}=0.165$ acre

${ }^{3}$ A 'traditional' farmer is a farmer engaged in agricultural production activities with the family as their main unit and self-sufficiency is their main purpose. Traditional farmers often have multiple jobs and are decentralized.
} 
and agricultural services, and insufficient support policy coverage (Xu, 2014). There are many reasons for these difficulties. First, even a perfect policy system must be put into practice to realize its value, and policy communication directly affects its implementation (Christen et al., 2015). However, most domestic studies focus on policy measures and do not pay attention to the channel of policy communication. Second, the formulation and implementation of support policies depend on the family farm construct (Stojcheska et al., 2016). However, domestic studies do not fully consider the preferences of family farms over support policy and so do not fully reflect the degree of acceptance by family farms of different support policies. Thus, the policies cannot properly encourage family farms.

Support policies based on the preferences of family farms can be provided after recognizing their heterogeneous preferences. Adamowicz et al. (1998) proposed the choice experiment method as an effective approach for eliciting such preferences. We focus on grain family farms (GFFs) and use the choice experiment method to examine support policy attributes and the attribute levels for the two dimensions of policy measures and policy communication channels. We eliminate ordering effects through warm-up questions for respondents and advance disclosure. We use the inferred attribute non-attendance (AN-A) method to process the attributes ignored by GFFs. Finally, we use a mixed logit model (MLM) and a latent class model (LCM) to analyze the preferences of GFFs for different support policies and how differences in GFFs' attributes correspond to different preference types, respectively.

This paper makes two main contributions. First, our comprehensive consideration of policy measures and policy dissemination channels makes up for the shortcomings of studies that ignore the policy communication channel. Second, this paper reveals the heterogeneity in the preferences of China's GFFs over support policies, analyzes the differences in the characteristics of family farms with different preferences, and proposes more feasible and targeted support policies.

\section{Experimental design and statistical description}

\subsection{Choice experiment design}

\section{- Attribute and level settings}

We use the choice experiment to describe the GFFs' support policies as a combination of attributes and to determine the state level of each attribute. To implement the choice experiment method, we reviewed the relevant literature and conducted interviews with grassroots agricultural department staff and GFFs to determine the initial support policy attributes and their levels. A choice experiment questionnaire for GFFs was designed to carry out the pre-investigation. Based on the outcome of the pre-investigation, we improved the choice experiment questionnaire with the help of nine agricultural policy research experts ${ }^{4}$. The following policy attributes and their levels were identified through these steps ${ }^{5}$.

Technical support is when the government provides technical guidance to GFFs through direct or indirect policy measures. Substantial technical support allows GFFs to master modern agricultural technology, achieve high production efficiency and cost savings, and reduce their energy consumption. Three levels of technical support (no technical support, medium technical support, and high technical support) were allowed for in accordance with the findings of Pan et al. (2016). There is no technical support when a grain family farm (GFF) engages in production and business operations according to experience or by following the example of others. Medium technical support is when a GFF obtains relevant technical guidance on the production process from an agricultural technology extension station or other institution. High technical support is

\footnotetext{
${ }^{4}$ The nine experts include five scholars and four staff members of the agricultural grassroots departments. The five scholars are from universities in Hebei, Henan, Anhui, Shandong and Jiangsu provinces, and are committed to related research. The four staff members of the agricultural grassroots departments are from Henan, Anhui, Shandong and Jiangsu provinces. The geographical distribution of the nine experts is relatively uniform and familiar with the local family farm support policies. It has a strong representativeness.

${ }^{5}$ In China, organizations and individuals directly engaged in agricultural production are tax-exempt and an agricultural insurance policy is applied to insurance companies. This paper does not regard tax incentives and agricultural insurance as attribute variables for family farm support policies.
} 
provided by agricultural technology extension staff to meet the specific needs of a GFF. China's agricultural technology extension system has serious problems, such as homogenization, insufficient investment, and inadequate high-quality talent. These problems hinder the effective implementation of technical support policies (Kong and Lou, 2012). Thus, the baseline of this attribute was no technical support.

Agricultural subsidies are the government's subsidies for the production and operation of GFFs. They play an important role in reducing production costs and raising incomes (Kirwan, 2009). In this study, agricultural subsidies refer to reasonable scale subsidies and agricultural support protection subsidies, including agricultural material comprehensive subsidies, direct subsidies for grain planting, and seed subsidies, but do not include agricultural machinery subsidies. We exclude agricultural machinery subsidies because they are meant for purchasing large-scale agricultural machinery. Large machinery is too expensive for most GFFs and few farms obtain such subsidies. Many farms do not know the exact amount of each subsidy they receive; they know the total amount of all subsidies they receive because subsidies are directly sent to the accounts of GFFs. Three levels of total agricultural subsidies $(103,153$, and 213 yuan/mu) were allowed for. One reason for selecting these values is that the minimum value of agricultural subsidies for farmers in the main grain producing areas is $43 \mathrm{yuan} / \mathrm{mu}$, the maximum value is $153 \mathrm{yuan} / \mathrm{mu}$, and the average value is $93 \mathrm{yuan} / \mathrm{mu}$ (Wang and Yang, 2014). Another reason for these values is that the reasonable scale subsidies per mu is 60 yuan per farmer for GFFs of 50 acres to 200 acres and 12,000 yuan per farmer for farms of more than 200 acres $^{6}$. Because the pre-survey found that the majority of GFFs had approximately $100 \mathrm{yuan} / \mathrm{mu}$ in total subsidies, the baseline of this attribute was 103 yuan/mu.

Credit support is the financial support provided by banks and other financial institutions to help farmers meet the large amount of financing needed for the development of GFFs. Credit support mitigates shortages of funds and increases the accumulation of material capital by family farms (Turvey and Xiong, 2017). Three levels of the credit support attribute (no credit support, 50,000 yuan, and 200,000 yuan) were allowed for, following the research of Zhang and Pan (2015). Current financial institutions provide credit support imperfectly and the 'fear of lending' phenomenon is prominent (Lan et al., 2015). Therefore, the baseline of this attribute was no credit support.

Educational training refers to training activities aimed at improving the entrepreneurial ability of farmers. Such training develops the decision-making capacity of farmers, improves the allocation of resources to GFFs, and increases incomes (Reimers and Klasen, 2013). Three levels of the educational training attribute (no educational training, decentralized educational training, and systematic educational training) were allowed for, following the research of Wang and Tian (2015) on the division of educational training. Farmers with no educational training rely on their own experience and skill in operation. Decentralized educational training is irregularly organized by the government for GFFs during the slack season or during critical stages. Systematic educational training is when the government regularly offers farmers' education and training adapted to their aptitudes and needs. Liu et al. (2015) argued that China's agricultural training of education is simple and repetitive and lacks effective incentives and restraint mechanisms. These deficiencies lead to a lack of enthusiasm by GFFs for such education and training. Thus, the baseline of this attribute was no educational training.

Land transfer support refers to policy adjustments that encourage family farms to obtain land by renting, exchanging, transferring, or some other means based on the choice of farmers and the collective ownership of the land. These policies ensure that GFFs achieve a modest scale by centralizing. The three levels of the land transfer support attribute (no land transfer support, medium land transfer support, and high land transfer support) were chosen based on the differences in the participation of the principal and the degree of government participation in land transfers. There is no land transfer support when family farms directly communicate with traditional farmers about rentals, shares, transfers, and other ways of obtaining land. Medium land transfer

\footnotetext{
${ }^{6}$ Shandong provincial finance department 'Our province is to improve the large grain farmer and the grain family farm subsidies.' Available at: http://czt.shandong.gov.cn/art/2015/10/26/art_21859_4156781.html
} 
support exists when the government functions as a bridge between traditional farmers and family farms when problems arise during land transfers. High land transfer support refers to the government integrating fragmented land parcels for unified management and transferring contiguous land to family farms. Land transfers involve the land rights and interests of multiple parties and are prone to disputes. Existing land transfer policies and laws are imperfect and there is no uniform land transfer price standard, causing the government difficulties in coordination and arbitration (Wei, 2009). Therefore, the baseline of this attribute was no land transfer support.

Policy communication channels refer to how GFFs obtain policy information. The spread of agricultural policies is closely linked to their implementation (Xie and Zhao, 2016). The dissemination of agricultural policy information relies on grassroots organizations, traditional media (television, radio, and newspapers), new media (the Weibo and WeChat public platforms), and other channels. Tan et al. (2005) argued that diversified communication channels are conducive to the effective communication of policy information. Thus, three levels of the policy communication channel attribute (single, general, and multiple channels) were allowed for. A single channel is when policy information is disseminated through traditional media. General channels refer to disseminating policy information through grassroots organizations and traditional media. Multiple channels involve the use of grassroots organizations, traditional media, and new media to disseminate policy information. Xie and Zhao (2016) reported that $75.77 \%$ of farmers obtain agricultural policy information from television. Therefore, the baseline of this attribute was a single channel.

Support for family farms aims to improve their profitability and their long-term capacity (Baležentis et al., 2014). Thus, the change in the profit of a GFF is the outcome variable of the support policy. Three levels of the change in profit attribute (unchanged, increase by $3 \%$, and increase by $6 \%$ ) were allowed for, following the survey of Gao et al. (2017a). China's family farms face an insufficient supply of financing, difficult land transfers, and many other issues, so a short-term rapid increase in profitability is difficult to achieve (Zhao et al., 2015). Therefore, the baseline of this attribute was unchanged.

\section{- Choice task design}

The total number of possible combinations of attribute levels is $2,187\left(3^{7}\right)$ in a full factorial design. It is unrealistic for farmers to compare and select from $\mathrm{C}^{2}{ }_{2,187}$ options. To achieve a manageable number of options, we used the approach of orthogonal optimality in the fractional factorial design, resulting in 12 profiles. The design exhibited a relatively good level of D-optimality, with D-efficiency of $91.4 \%$. The 12 choice sets were divided into three blocks with four choice sets each. The respondents were randomly distributed to one of these blocks. Each randomly selected farmer faced four choice cards. Farmers in each choice set were asked to choose between two alternative policy combinations of options and a status quo option.

To reduce the influence of ordering effects on the policy preferences of GFFs, four choice cards were randomly ranked in each questionnaire. In addition, before the formal questionnaire began, the farmers were told the meaning of each attribute and state level and were provided with a sample choice scene as a warm-up question to familiarize them with the experimental process.

In addition, in order to eliminate the influence of ordering effects on GFFs' policy preferences, firstly, the four choice experiment cards are randomly ranked in each experimental questionnaire, and the ordering of the attributes of each choice experimental card is also random. Secondly, during the formal investigation, the investigator 'disclosed' the key information of the experiment card to the GFFs in advance, that is, detailed description of the specific meanings, choice times and other related information of each attribute and its status level, and carefully answered the questions raised by the GFFs. Finally, in order to 'warm up', the investigator provides the GFFs with an example of a choice. To ensure that the real choice of GFFs is not affected, this example is not included in the formal selection of the experimental questionnaire, and is only for the GFFs to get familiar with the selection of experimental cards and the experimental process. 


\subsection{Data}

\section{- Data collection}

After considering regional development imbalances and policy differences across China, the Huang-huai-hai Plain (which includes Hebei, Henan, Shandong, Anhui, and Jiangsu provinces) was selected as the location for the field research. The main reasons for this selection were that: (1) the five provinces have similar geographical and natural conditions and are the main provinces for China's food production; and (2) the number of family farms in these five provinces has grown significantly, with more than 10,000 households registered in the business sectors (Gao et al., 2019b).

The survey was conducted in two stages. The first stage was the pre-survey. Ten to twenty GFF households were selected in each province using typical sampling methods. Face-to-face interviews were conducted to understand the local policies supporting GFFs, the operating conditions of farms, and their demands for policies. The results of this survey were used as the basis for the design of the experimental attributes and questionnaire. The questionnaire was administered in the second stage. An investigative team of trained college students and postgraduates conducted a formal investigation of the five provinces between December 2016 and February 2017. There are 74 prefecture-level cities in these five provinces. Based on the rural per capita income level of cities, the investigative team equidistantly extracted three counties in each prefecturelevel city. Three GFFs were randomly selected in each county for investigation. The investigators guided the selected family farms though the questionnaire to guarantee quality. In total, 666 questionnaires were distributed and 570 questionnaires were fully completed after eliminating contradictory and incomplete information questionnaires, for a valid response rate of $85.6 \%$.

\section{- Variable selection and measurement}

A large number of studies have shown that farmers' characteristics (Breustedt et al., 2008), resource endowment characteristics (Van de Gucht et al., 2017) and psychological cognition characteristics (Pan et al., 2016; Schulz et al., 2014) are the main factors affecting the change of GFFs' profitability. Therefore, this paper discusses the factors that affect the profitability of GFFs from three dimensions: farmers' characteristics, resource endowment characteristics and psychological cognitive characteristics.

In terms of farmer characteristics, age exerts a significantly negative effect on the profit of a GFFs (Roy et al., 2014). A farmer's education level significantly and positively affects the performance of the family farm (Panda, 2015). Younger and better educated farmers are more likely to run profitable farms.

In terms of resource endowment, households with fewer laborers often incur high labor costs, which significantly reduce profits (Zhu et al., 2014). A reasonable scale of operation is also key to agricultural development (Huang, 2014). A reasonable scale of operation allows a family farm to realize the optimal allocation of various production factors. Therefore, facing the same combination of policy attributes, GFFs with more family laborers and with more appropriate scales of operation should be more profitable.

In terms of psychological cognition, the acceptance and selection of support policies by family farms are affected by their cognition. Facing the same combination of policy attributes, farmers with high degrees of cognition of support policies are more likely to improve their farms' profitability by using policies flexibly (Yin et al., 2017).

We choose the age of farmers, the educational level of farmers, the number of family laborers, how reasonable the land scale is, and the degree of cognition of support policy as the factors influencing changes in the profits of GFFs. A farmer's age is measured as the farmer's age in 2016. A farmer's educational level is measured by their years of education. The number of family laborers is defined as the number of people in the family 
with the ability to work. The land scale is measured as: (1) unreasonable; (2) neutral; or (3) reasonable. The cognitive degree of support policy is measured as: (1) low; (2) neutral; or (3) high.

\section{- Statistical description}

As shown in Table 1, the average age of the farmers sampled was 42.4 years, and $96.0 \%$ were under the age of 60 . This is consistent with the prevalence of middle-aged and young farmers in China. Farmers had 8.6 years of education on average, and $71.1 \%$ had between 8 and 12 years of education. This is consistent with the middle school education level of the labor force. The average number of family laborers was 3.1 and $94.2 \%$ of the households had between two and five family laborers, consistent with the characteristics of China's rural family population. The average land scale was 2.0 , and the land scale of $82.5 \%$ of the farms was rated as 'neutral' or 'reasonable.' This reflects that most Chinese family farms are of a reasonable scale. The age of farmers, their level of education, the number of family laborers, and the land scale reflect that the survey sample was representative of the target population. The average cognitive degree of support policy was 2.1 , and $86.3 \%$ of GFFs had a 'neutral' or 'high' cognitive level. GFFs were expected to exhibit a high level of understanding of support policies.

\section{Model}

\subsection{Mixed logit model and latent class model}

Individuals choose a combination of policy attributes to maximize their own utility (Lancaster, 1966). If GFF $i$ chooses the policy attribute combination $j$ out of possible policy attribute combinations $J$, their resulting utility is:

$$
U_{i j}=V_{i j}+\varepsilon_{i j}=\beta_{i}^{\prime} X_{i j}+\varepsilon_{i j} \quad \beta_{i} \sim N(b, W)
$$

where $V_{i j}$ is the definite part, $\varepsilon_{i j}$ is the random part (a stochastic error term), and $X_{i j}$ represents the attribute vector of the GFF. $\mathrm{B}_{\mathrm{i}}$ is the utility score vector, which is the individual preference coefficient of GFF $i$ and is assumed to be distributed according to a normal distribution with mean $b$ and covariance $W$.

The adopted model is different because of varying assumptions regarding the stochastic part $\varepsilon_{i j} \varepsilon_{i j}$ is assumed to be independently and identically drawn from the I-type extreme distribution. Hence, the probability that GFF $i$ selects the support policy combination $j$ instead of the combination $m$ can be represented by an MLM as:

$$
P_{i j}=\operatorname{Pr}\left(U_{i j}>U_{i m}, \forall j \neq m\right)=\int \frac{\exp \left(\beta_{i}^{\prime} X_{i j}\right)}{\sum_{m \in J} \exp \left(\beta_{i}^{\prime} X_{i m}\right)} f(\beta \mid \theta) d \beta
$$

where $f(\beta \mid \theta)$ is the probability density of $\beta$, which is a random variable that follows the $f(\beta \mid \theta)$ distribution, and $\theta$ is the parameter that describes this distribution.

Table 1. Descriptive statistics.

\begin{tabular}{llrl}
\hline Variable & Description & Mean & Std. deviation \\
\hline Age & farmer's actual age in 2016 & 42.4 & 7.4 \\
Education & farmer's years of education & 8.6 & 2.6 \\
Family labor & number of family laborers & 3.1 & 0.7 \\
Reasonable degree of land scale & 1 = unreasonable; 2 = neutral; 3 = reasonable & 2.0 & 0.6 \\
Cognitive degree of support policy & 1 = low; 2 = neutral; 3 = high & 2.1 & 0.6 \\
\hline
\end{tabular}


If $f(\beta \mid \theta)$ is discrete, then Equation 2 can be further transformed into a LCM to determine the classes of different GFFs and solve the problem of arbitrarily dividing classes (Hou, 2018; Krishnakumar and ChanHalbrendt, 2010; Wen and Lai, 2011). $N$ GFFs can be divided into $S$ latent classes with family farms with the same or similar preferences put into the same class. Then, the probability GFF $i$ chooses support policy combination $j$ is:

$$
P_{i j}=\sum_{s=1}^{S} \frac{\exp \left(\beta_{s} X_{i j}\right)}{\sum_{m \in J} \exp \left(\beta_{s} X_{i m}\right)} R_{i s}
$$

where $\beta_{s}$ is the parameter vector of GFFs in class $s$ and $R_{i s}$ is the probability GFF $i$ is in latent class $s$, which is:

$$
R_{i s}=\frac{\exp \left(\mu_{s} Z_{i}\right)}{\sum_{r \in S} \exp \left(\mu_{r} Z_{i}\right)}
$$

where $r$ is the $r^{\text {th }}$ latent class, $\mu_{s}$ is the parameter vector of GFFs in latent class $s$, and $Z_{i}$ represents a series of characteristic vectors that influence which latent class GFF $i$ falls into.

The MLM reveals GFFs' heterogeneous preferences over support policies. The LCM divides GFFs into several classes with heterogeneous preferences. Combining these two methods allows for a deeper understanding of the definite part $V_{i j}$ of GFFs' preferences. The attributes, outcomes, characteristics of GFFs, and their interaction terms are introduced into the equation of the observable utility part $V_{i j}$ as:

$$
V_{i j}=A S C+\beta_{p r o} P R O+\sum_{k=1}^{K} \beta_{j k} x_{j k}+\sum_{k=1}^{K} \tau_{i k} x_{j k}+\sum_{h=1}^{H} a_{h}\left(S_{i h} \times A S C\right)
$$

where $A S C$ is the alternative specific constant, which measures the effects of unobservable factors on the policy choice of GFF $i$. This constant excludes the policy attributes and the characteristics of GFFs. In each choice experiment card, when GFF $i$ chooses the current policies, ASC is taken to be 1 . When the family farm chooses another policy combination, $A S C$ is 0 . A negative value of $A S C$ shows that the GFF is more willing to choose the policy combination than to maintain the status quo. PRO is the outcome variable, the change in the profit of the GFF.

$\beta_{\text {pro }}$ is the coefficient on $P R O . \beta_{j k}$ is the coefficient on the $k^{\text {th }}(k=1,2, \ldots, 6)$ attribute variable $x_{j k}$ for policy attribute combination $j . \tau_{i k}$ measures the difference between the individual coefficient for GFF $i$ and the population mean coefficient $\beta_{j k}$ for attribute variable $x_{j k}$. This value refers to the deviation between the marginal utility of an individual family farm that selects a support policy combination and the marginal utility of the average family farm that selects this support policy combination. When $\tau_{i k}$ is statistically significant, there is heterogeneity in the preferences of GFFs for that support policy combination. $S_{i h}$ is the $h^{\text {th }}$ characteristic variable of GFF $i . \alpha_{h}$ is the coefficient on the interaction term between the characteristic variable and $A S C$ and measures the influence of that characteristic on the policy choices of GFFs. In parameter estimation, we use hierarchical Bayesian estimation to directly estimate the likelihood function ${ }^{7}$ to avoid the optimal solution instability problem caused by different initial points and to ensure unbiased estimation results under more relaxed conditions (Byun and Lee, 2017; Gao et al., 2018).

\subsection{Inferred attribute non-attendance method}

The choice experiment method is based on rational preferences. In practice, because of limited cognitive ability or high information load, interviewees ignore the influence of individual attributes on their choice decision to simplify their decision-making process. This is called the AN-A problem (Hensher et al., 2005). To reduce the estimation bias caused by the irrational preferences of interviewees and improve the validity of the model, information processing should be applied to the attributes neglected by interviewees (Scarpa

\footnotetext{
${ }^{7}$ Because of space limitations, the details of the hierarchical Bayesian method are not included.
} 
et al., 2009). The common methods of information processing are stated AN-A and inferred AN-A. The inferred AN-A method performs better (Bir et al., 2018; Gao et al., 2018; Hess and Hensher, 2010; Nguyen et al., 2015; Ortega and Ward, 2016), so we follow Hess and Hensher (2010) in adopting the inferred AN-A method. We set an optimal threshold value based on the estimated value of the model parameters. Any attribute with a coefficient of variation above the optimal threshold value of $1.7^{8}$ is ignored; the attribute is not involved in the model estimation. The coefficient of variation is the ratio of the conditional standard deviation to the conditional mean, which can be expressed as:

$$
C . V_{i m}=E\left[\sigma_{i m} \mid \text { data }_{i}\right] / E\left[\beta_{i m} \mid \text { data }_{i}\right]
$$

where $E\left[\sigma_{i m} \mid d a t a_{i}\right]$ is the conditional standard deviation of attribute $m$ for GFF $i$ and $E\left[\sigma_{i m} \mid d a t a_{i}\right]$ is the conditional mean of attribute $m$ for GFF $i$.

\section{Results and discussion}

\subsection{Results of information processing strategies and discussion}

We use the inferred AN-A method to deal with the AN-A problem. As shown in Table 2, education training AN-A affects the most households (4.74\% of households) and agricultural subsidy AN-A affects the fewest households (1.93\% of households). There are several potential reasons for this. First, family farmers have better agricultural skills, management experience, and learning abilities than traditional farmers. Therefore, the importance of educational training for GFFs is relatively low. Second, the periodicity and seasonality of production mean that GFFs have high cash flow costs and agricultural subsidies alleviate financial pressures (Vercammen, 2007). Thus, grain family farmers place great importance on agricultural subsidies.

\subsection{Results of mixed logit model and discussion}

The MLM is constructed using the data processed by the inferential AN-A method. The estimation results are shown in Table 3. The coefficient on ASC is significant and negative, which indicates that GFFs are more likely to change the status quo and choose a more appropriate combination of policy attributes.

Several of the mean coefficients are significant and positive, including those on decentralized educational training and general channels. The profits of GFFs increase when they receive medium or high technical support, more agricultural subsidies and credit support, systematic educational training, medium or high land transfer support, and access to policy information through multiple channels. This supports existing findings. Yang and An (2002) found that technical support, formal education, and training are key to increasing family farms profits. Manjunatha et al. (2013) pointed out that family farms with more concentrated land holdings and large-scale family farms have higher profits than other farms. Kirwan (2009) argued that agricultural

\footnotetext{
${ }^{8}$ We use the iterative search method proposed by Hess and Hensher (2010) (which ensures the existence and effectiveness of the optimal threshold) to determine the optimal threshold value of 1.7. Because of space limitations, details are omitted.
}

Table 2. Processing results of inferred attribute non-attendance method.

\begin{tabular}{lll}
\hline \multirow{2}{*}{ Attribute } & \multicolumn{2}{l}{ Inferred attribute non-attendance } \\
\cline { 2 - 3 } & Respondents & Rate (\%) \\
\hline Technical support & 23 & 4.04 \\
Agricultural subsidy & 11 & 1.93 \\
Credit support & 18 & 3.16 \\
Educational training & 27 & 4.74 \\
Land transfer support & 15 & 2.63 \\
Policy communication channel & 17 & 2.98 \\
\hline
\end{tabular}


Table 3. Mixed logit model results.

\begin{tabular}{|c|c|c|}
\hline Variable & Coefficient ${ }^{1}$ & Std. error \\
\hline \multicolumn{3}{|l|}{ Mean coefficient } \\
\hline Constant $(A S C)$ & $-0.715^{* * *}$ & 0.258 \\
\hline Medium technical support & $0.893^{* *}$ & 0.386 \\
\hline High technical support & $1.006^{* *}$ & 0.464 \\
\hline Agricultural subsidy & $1.364^{* * *}$ & 0.380 \\
\hline Credit support & $1.085^{* *}$ & 0.539 \\
\hline Decentralized educational training & 0.279 & 0.263 \\
\hline Systemic educational training & $0.356^{* *}$ & 0.160 \\
\hline Medium land transfer support & $0.594^{* *}$ & 0.292 \\
\hline High land transfer support & $0.527^{* *}$ & 0.243 \\
\hline General channels & 0.473 & 0.457 \\
\hline Multiple channels & $0.564^{* *}$ & 0.240 \\
\hline Change in profit & $0.024^{* *}$ & 0.011 \\
\hline$A S C \times$ Age & 0.063 & 0.196 \\
\hline$A S C \times$ Education & $-0.032^{* *}$ & 0.015 \\
\hline$A S C \times$ Family labor & -0.039 & 0.032 \\
\hline$A S C \times$ Neutral land scale & -0.055 & 0.039 \\
\hline$A S C \times$ reasonable land scale & $-0.071^{* * *}$ & 0.026 \\
\hline$A S C \times$ neutral cognitive degree & -0.072 & 0.054 \\
\hline$A S C \times$ high cognitive degree & $-0.058^{* * *}$ & 0.020 \\
\hline \multicolumn{3}{|l|}{ Standard deviation coefficient } \\
\hline Medium technical support & $0.201^{*}$ & 0.112 \\
\hline High technical support & $0.315^{* *}$ & 0.135 \\
\hline Agricultural subsidy & $0.524^{*}$ & 0.312 \\
\hline Credit support & $0.425^{* *}$ & 0.180 \\
\hline Decentralized educational training & $0.356^{* *}$ & 0.168 \\
\hline Systemic educational training & $0.499^{* *}$ & 0.222 \\
\hline Medium land transfer support & $0.243^{*}$ & 0.130 \\
\hline High land transfer support & $0.312^{* *}$ & 0.155 \\
\hline General channels & $0.206^{*}$ & 0.117 \\
\hline Multiple channels & $0.427^{* *}$ & 0.180 \\
\hline \multicolumn{3}{|l|}{ Model statistics } \\
\hline Log-likelihood & $-4,176.903$ & \\
\hline McFadden Pseudo- $\mathrm{R}^{2}$ & 0.329 & \\
\hline
\end{tabular}

subsidies benefit family farms by increasing agricultural production. Petrini et al. (2016) argued that credit support promotes the development of family farms and that diversified channels of policy communication enhance family farms' cognition of support policies, helping farmers to use support policies flexibly to maximize their benefits. The mean coefficient on agricultural subsidies, credit support, and high and medium technical support are significantly greater than those on other policy attribute variables. This shows that GFFs want to be supported by the government in financing and technology.

The standard deviation coefficients of all policy attribute variables are significant. This indicates that there is heterogeneity in the preferences of GFFs for technical support, agricultural subsidies, credit support, educational training, land transfer support, and the policy communication channel. 
The coefficients on the interactions terms between $A S C$ and the variables measuring the education level of farmers, the reasonability of the land scale, and the degree of cognition of support policies are statistically significant and negative. The negative signs of these interactions indicate that higher levels of education, more reasonable land scales, and higher degrees of cognition of support policies positively influence farms' profits. These results make sense for several reasons. First, farmers with more education and a stronger ability to make decisions are better at integrating resources and improving their farms' profits (Reimers and Klasen, 2013). Second, the size of a GFF impacts its ability to achieve the optimal allocation and efficient use of the natural and social factors of production (Manjunatha et al., 2013). Third, farmers with a high level of understanding of policy support make full use of the support policies related to their own interests. Thus, they greatly enjoy the benefits of the policy. The interaction between $A S C$ and the age of the farmer is insignificant, which may relate to the fact that middle-aged and young farmers constitute the majority of China's grain family farmers. The interaction between $A S C$ and the number of family laborers is also insignificant. The probable reason for this is that the contribution rate of the traditional factors of grain production has adjusted, with a gradual decrease in the impact of family laborers on the profitability of GFFs resulting from agricultural modernization in China (Ye and Tuo, 2015).

\subsection{Results of latent class model and discussion}

We use the LCM to further analyze the policy preferences of different types of GFFs. Most studies use the Akaike information criterion or the Bayesian information criterion (BIC) to measure model fitting effects and to test the adaptability of the model (Orea and Kumbhakar, 2004). Ruto and Garrod (2009) found that the BIC effectively prevents the model from over-fitting when the number of observations is large. Thus, we use the BIC for the classification basis of the LCM. Table 4 shows that the BIC value is optimized by splitting GFFs into four types. The GFFs are divided into four distinct types based on their utility from policy attributes. Of the six policy attributes used in this study, two policy attributes belong to a single category. The final LCM estimation is shown in Table 5.

The estimated coefficients on agricultural subsidies and credit support are relatively large for Class 1 farms, indicating that this type of GFFs has a strong demand for funds. We label these farms as 'finance preference' types. This class accounts for $43.2 \%$ of GFFs and it is the largest class. This is consistent with the results of the MLM that GFFs desire agricultural subsidies and credit support. In addition, young farmers tend to be short on funds and have a more urgent demand for funds than older farmers (Oluwasola and Alimi, 2008). Accordingly, age exerts a significant negative effect on the probability that a farm falls into Class 1 .

The estimated coefficients on medium technical support, high technical support, decentralized educational training, and systematic educational training are relatively large for Class 2 farms. As these GFFs have a strong preference for knowledge and technology, we label these farms as 'knowledge and technology preferences' types; $28.5 \%$ of farms fall into this category. Farmers with a higher level of education and degree of cognition of support policies have a better understanding of the importance of knowledge and technology in the development of family farms (Reimers and Klasen, 2013). Accordingly, the level of education and

Table 4. Criteria used for setting the optimal class number.

\begin{tabular}{ll}
\hline Number of classes & Bayesian information criterion \\
\hline 2 & $3,517.423$ \\
3 & $3,462.210$ \\
4 & $3,225.083$ \\
5 & $3,364.759$ \\
6 & $3,497.655$ \\
7 & $3,633.787$ \\
\hline
\end{tabular}


Table 5. Latent class model results.

\begin{tabular}{|c|c|c|c|c|c|c|c|c|}
\hline \multirow[t]{2}{*}{ Variable } & \multicolumn{2}{|c|}{$\begin{array}{l}\text { Class } 1 \text { 'finance } \\
\text { preference' }\end{array}$} & \multicolumn{2}{|c|}{$\begin{array}{l}\text { Class } 2 \text { 'knowledge } \\
\text { and technology } \\
\text { preferences' }\end{array}$} & \multicolumn{2}{|c|}{$\begin{array}{l}\text { Class } 3 \text { 'land } \\
\text { transfer } \\
\text { preference' }\end{array}$} & \multicolumn{2}{|c|}{$\begin{array}{l}\text { Class } 4 \text { 'policy } \\
\text { information } \\
\text { preference' }\end{array}$} \\
\hline & Coefficient $^{1}$ & $\begin{array}{l}\text { Std. } \\
\text { error }\end{array}$ & Coefficient & $\begin{array}{l}\text { Std. } \\
\text { error }\end{array}$ & Coefficient & $\begin{array}{l}\text { Std. } \\
\text { error }\end{array}$ & Coefficient & $\begin{array}{l}\text { Std. } \\
\text { error }\end{array}$ \\
\hline Constant (ASC) & $-0.651^{* * *}$ & 0.217 & $-0.477^{* *}$ & 0.225 & $-0.403^{* *}$ & 0.186 & $-0.545^{* *}$ & 0.263 \\
\hline $\begin{array}{l}\text { Medium technical } \\
\text { support }\end{array}$ & 0.604 & 0.489 & $2.082^{* * *}$ & 0.641 & 0.485 & 0.328 & 0.543 & 0.370 \\
\hline High technical support & $0.836^{* *}$ & 0.357 & $2.535^{* *}$ & 1.015 & 0.326 & 0.259 & 0.345 & 0.253 \\
\hline Agricultural subsidy & $1.457^{* * *}$ & 0.488 & $1.163^{* *}$ & 0.542 & $0.749^{* *}$ & 0.364 & $0.866^{* *}$ & 0.386 \\
\hline Credit support & $2.392^{* * *}$ & 0.865 & $0.914^{* *}$ & 0.422 & $0.436^{* *}$ & 0.202 & $0.627^{*}$ & 0.351 \\
\hline $\begin{array}{l}\text { Decentralized } \\
\text { educational training }\end{array}$ & 0.549 & 0.383 & $1.547^{* * *}$ & 0.555 & 0.397 & 0.267 & 0.464 & 0.421 \\
\hline $\begin{array}{l}\text { Systemic educational } \\
\text { training }\end{array}$ & 0.620 & 0.489 & $1.703^{* *}$ & 0.719 & 0.331 & 0.316 & $0.432^{* *}$ & 0.201 \\
\hline $\begin{array}{l}\text { Medium land transfer } \\
\text { support }\end{array}$ & $0.533^{*}$ & 0.302 & 0.364 & 0.292 & $1.086^{* * *}$ & 0.335 & 0.339 & 0.225 \\
\hline $\begin{array}{l}\text { High land transfer } \\
\text { support }\end{array}$ & 0.716 & 0.493 & 0.295 & 0.244 & $1.557^{* *}$ & 0.765 & 0.194 & 0.187 \\
\hline General channels & $0.532^{* *}$ & 0.238 & 0.463 & 0.345 & $0.561^{* *}$ & 0.241 & $1.304^{* * *}$ & 0.428 \\
\hline Multiple channels & $0.497^{*}$ & 0.267 & $0.561^{* *}$ & 0.241 & $0.467^{* *}$ & 0.203 & $2.026^{* * *}$ & 0.624 \\
\hline Change in profit & $0.797^{* * *}$ & 0.281 & $0.525^{* *}$ & 0.238 & $0.326^{* *}$ & 0.135 & $0.311^{*}$ & 0.159 \\
\hline Age & $-0.082^{* * *}$ & 0.027 & -0.026 & 0.020 & 0.057 & 0.045 & $-0.055^{* *}$ & 0.024 \\
\hline Education & 0.320 & 0.236 & $0.693^{* * *}$ & 0.243 & 0.286 & 0.196 & 0.512 & 0.415 \\
\hline Family labor & 0.361 & 0.273 & 0.322 & 0.221 & $0.974^{* *}$ & 0.414 & 0.155 & 0.124 \\
\hline $\begin{array}{l}\text { Reasonable degree of } \\
\text { land scale }\end{array}$ & 0.256 & 0.180 & 0.469 & 0.354 & 0.433 & 0.285 & 0.362 & 0.258 \\
\hline $\begin{array}{l}\text { Cognitive degree of } \\
\text { support policy }\end{array}$ & 0.414 & 0.477 & $0.758^{* *}$ & 0.350 & 0.642 & 0.402 & $0.533^{* *}$ & 0.250 \\
\hline Share (\%) & $43.2 \%$ & & $28.5 \%$ & & $15.4 \%$ & & $12.9 \%$ & \\
\hline
\end{tabular}

the degree of cognition of support policies exert a significantly positive effect on the probability a farm falls into Class 2.

The estimated coefficients on medium land transfer support and high land transfer support are relatively large for Class 3 farms. GFFs in this class are 'land transfer preference' type and account for $15.4 \%$ of farms. GFFs with more family laborers have a stronger desire to expand their scale, for which land transfers are required (Deininger and Jin, 2005). Accordingly, the number of family laborers has a significantly positive effect on the probability that a GFF is in Class 3.

The estimated coefficients on general channels and multiple channels for Class 4 farms are relatively large. These family farms are highly sensitive to policy information and so are labeled as 'policy information preference' types. They account for $12.9 \%$ of farms. Young farmers are more adept at using a wide range of communication channels than older farmers, allowing them to quickly obtain comprehensive and detailed policy information. Accordingly, age has a significantly negative effect on the probability that a farm falls into Class 4. Moreover, farms with a higher degree of cognition of support policies are more concerned about policy information. As seen in Table 5, the degree of cognition of support policies has a significantly positive influence on the probability of falling into Class 4 . 


\section{Conclusions and policy implications}

This study uses the choice experiment method with 570 GFFs located in the Huang-huai-hai Plain and determine various support policy attributes and the attribute levels for the two dimensions of policy measures and policy communication channels. Ordering effects are eliminated by warming up subjects in advance and proper information disclosure. The inferred AN-A method is used to process attributes ignored by the GFFs. This paper then uses a MLM to analyze GFFs' preferences over different support policies and analyzes which characteristics of GFFs are associated with which preference types through a LCM.

We draw three main conclusions from our results. First, the preferences of GFFs over support policies are heterogeneous, especially for agricultural subsidies, credit support, and technical support (the mean coefficient is greater than 0.8), but communication channel (the mean coefficient is greater than 0.5) preferences cannot be ignored. Second, with the same combination of policy attributes, it is possible to increase the profits of GFFs headed by highly educated farmers, those with a reasonable land scale, and those with a high degree of cognition of support policies. Third, GFFs can be divided into four types depending on their policy preferences: finance preference types (43.2\%), knowledge and technology preference types (28.5\%), land transfer preference types $(15.4 \%)$, and policy information preference types $(12.9 \%)$.

This study has four policy implications. First, support policies should focus on agricultural subsidies, credit support, and technical support as this will meet the strong demands of GFFs for funds and technology. Second, following the principle that 'the first rich farmers should help other farmers get rich,' farmers with high education levels, reasonable land scales, and high degrees of cognition of support policy should be supported first. These features allow the government to carry out targeted policy support. Third, the government should establish a precise combination of targeted poverty alleviation to enable GFFs with different policy preferences to be properly supported and to maximize the utility of support policies. Fourth, the government should promote the development of diversified policy communication methods combining grassroots organizations, traditional media, and new media to improve GFFs' satisfaction with and awareness of beneficial farming policies.

\section{Acknowledgements}

This work was supported by the National Natural Science Foundation of China (Grant: 71803096); the Humanity and Social Science of Ministry of Education of China (Grant: 18YJA790024) and the Shandong Province Natural Science Foundation of China (Grant: ZR2018MG009).

\section{References}

Adamowicz, W., P. Boxall, M. Williams and J. Louviere. 1998. Stated preference approaches for measuring passive use values: choice experiments and contingent valuation. American Journal of Agricultural Economics 80(1): 64-75.

Baležentis, T., I. Kriščiukaitienė and A. Baležentis. 2014. A nonparametric analysis of the determinants of family farm efficiency dynamics in Lithuania. Agricultural Economics 45(5): 589-599.

Barnes, A., L.A. Sutherland, L. Toma, K. Matthews and S. Thomson. 2016. The effect of the Common Agricultural Policy reforms on intentions towards food production: evidence from livestock farmers. Land Use Policy 50(4): 548-558.

Bir, C., A.M. Cummins, N.O. Widmar and C.A. Wolf. 2018. Willingness to pay estimates informing agribusiness decision making: a cautionary tale. International Food and Agribusiness Management Review 21: 865-882.

Botterill, L.C. 2016. Agricultural policy in Australia: deregulation, bipartisanship and agrarian sentiment. Australian Journal of Political Science 51(4): 667-682. 
Breustedt, G., J. Müller-Scheeßel and U. Latacz-Lohmann. 2008. Forecasting the adoption of GM oilseed rape: evidence from a discrete choice experiment in Germany. Journal of Agricultural Economics 59(2): 237-256.

Byun, H. and C.Y. Lee. 2017. Analyzing Korean consumers' latent preferences for electricity generation sources with a hierarchical Bayesian logit model in a discrete choice experiment. Energy Policy 105: 294-302.

Christen, B., C. Kjeldsen, T. Dalgaard and J. Martin-Ortega. 2015. Can fuzzy cognitive mapping help in agricultural policy design and communication? Land Use Policy 45: 64-75.

Deininger, K. and S.Q. Jin. 2005. The potential of land rental markets in the process of economic development: evidence from China. Journal of Development Economics 78(1): 241-270.

Du, Z.X. and W.D. Xiao. 2014. The actual state and policy support for the development of family farms: in the perspective of international experience. Reform 6: 39-51.

Gao, Y., D.Y. Zhao, L.L. Yu and H.R. Yang. 2019a. Duration analysis on the adoption behavior of green control techniques among Chinese farms. Environmental Science and Pollution Research 26(7): 6319-6327.

Gao, Y., P. Li, L. Wu, J. Lu, L. Yu and S. Yin. 2018. Support policy preferences of for-profit pest control firms in China. Journal of Clean Production 181: 809-818.

Gao, Y., X. Zhang, J. Lu, L. Wu and S. Yin. 2017b. Adoption behavior of green control techniques by family farms in China: evidence from 676 family farms in Huang-huai-hai plain. Crop Protection 99: 76-84.

Gao, Y., X. Zhang, L. Wu, S. Yin and J. Lu. 2017a. Resource basis, ecosystem and growth of grain family farm in China: based on rough set theory and hierarchical linear model. Agricultural Systems 154: 157-167.

Gao, Y., Z.H. Niu, H.R. Yang and L.L. Yu. 2019b. Impact of green control techniques on family farms' welfare. Ecological Economics 161: 91-99.

Graeub, B.E., M.J. Chappell and H. Wittman. 2015. The state of family farms in the world. World Development 15(6): 1-15.

Hensher, D.A., N. Shore and K. Train. 2005. Households' willingness to pay for water service attributes. Environmental and Resource Economics 32(4): 509-531.

Hess, S. and D.A. Hensher. 2010. Using conditioning on observed choices to retrieve individual-specific attribute processing strategies. Transportation Research Part B: Methodological 44(6): 781-790.

Hoppe, R.A. and D.E. Banker. 2010. Family farm report: structure and finances of U.S. farms. USDA, Economic Research Service, Washington, DC, USA.

Hou, B. 2018. Research on consumer preference and public policy of traceable food. Social Sciences Academic Press, Beijing, China.

Huang, P.C.C. 2014. Is 'family farms' the way to develop Chinese agriculture? Rural China 11(2): 189-221.

Kihl, Y.W. 1982. Farm structure and rural policy in Japan. Food Policy 7(4): 332-336.

Kirwan, B.E. 2009. The incidence of U.S. agricultural subsidies on farmland rental rates. Journal of Political Economy 117(1): 138-164.

Kong, X. and D. Lou. 2012. Comparison among different countries agricultural technology extension systems, current situations and China's countermeasures. Reform 1: 12-23.

Krishnakumar, J. and C. Chan-Halbrendt. 2010. Consumer preferences for imported Kona coffee in South India a latent class analysis. International Food and Agribusiness Management Review 13: 97-116.

Lancaster, K.J. 1966. A new approach to consumer theory. Journal of Political Economy 74(2): 132-157.

Lan, Y., M. Zhou and Z. Yi. 2015. Research on financial support of family farm in China. Journal of Agrotechnical Economics 6: 48-56.

Liu, J., C. Chen and H. Xie. 2015. An investigation on the current status of farmers' education and training. Educational Research 2: 123-129.

Lv, H. and Y. Zhu. 2015. Research on the development mode of the family farm: based on the quantitative analysis of the questionnaire: case of Ningbo city, Zhejiang province. Issues in Agricultural Economy 4: 19-26.

Manjunatha, A.V., A.R. Anik, S. Speelman and E.A. Nuppenau. 2013. Impact of land fragmentation, farm size, land ownership and crop diversity on profit and efficiency of irrigated farms in India. Land Use Policy 31(31): 397-405.

McFadden, T. and M. Gorman. 2016. Exploring the concept of farm household innovation capacity in relation to farm diversification in policy context. Journal of Rural Studies 46: 60-70. 
Medina, G., C. Almeida, E. Novaes, G.J. Evandro and B. Pokorny. 2015. Development conditions for family farming: Lessons from Brazil. World Development 74: 386-396.

Nguyen, T., J. Robinson, S. Kaneko and N. The-Chinh. 2015. Examining ordering effects in discrete choice experiments: a case study in Vietnam. Economic Analysis and Policy 45: 39-57.

Oluwasola, O. and T. Alimi. 2008. Determinants of agricultural credit demand and supply among small-scale farmers in Nigeria. Outlook on Agriculture 37(3): 185-193.

Orea, L. and S. Kumbhakar. 2004. Efficiency measurement using a latent class stochastic frontier model. Empirical Economics 29(1): 169-183.

Ortega, D.L. and P.S. Ward. 2016. Information processing strategies and framing effects in developing country choice experiments: results from rice farmers in India. Agricultural Economics 47(5): 493-504.

Pan, D., G. Zhou, N. Zhang and L. Zhang. 2016. Farmers' preferences for livestock pollution control policy in China: a choice experiment method. Journal of Clean Production 131: 572-582.

Panda, S. 2015. Farmer education and household agricultural income in rural India. International Journal of Social Economics 42(6): 514-529.

Petrini, M.A., J.V. Rocha, J.C. Brown and R.C. Bispo. 2016. Using an analytic hierarchy process approach to prioritize public policies addressing family farming in Brazil. Land Use Policy 51: 85-94.

Reimers, M. and S. Klasen. 2013. Revisiting the role of education for agricultural productivity. American Journal of Agricultural Economics 95(1): 131-152.

Roy, R., N.W. Chan, R. Rainis. 2014. Rice farming sustainability assessment in Bangladesh. Sustain Science 9(1): 31-44.

Ruto, E. and G. Garrod. 2009. Investigating farmers' preferences for the design of agri-environment schemes: a choice experiment approach. Journal of Environmental Planning and Management 52(5): 631-647.

Scarpa, R., T.J. Gilbride, D. Campbell and D.A. Hensher. 2009. Modelling attribute non-attendance in choice experiments for rural landscape valuation. European Review of Agricultural Economics 36(2): 151-174.

Schulz, N., G. Breustedt and U. Latacz-Lohmann. 2014. Assessing farmers' willingness to accept 'Greening': insights from a discrete choice experiment in Germany. Journal of Agricultural Economics 65(1): 26-48.

Stojcheska, A.M., A. Kotevska, N. Bogdanov and A. Nikolić. 2016. How do farmers respond to rural development policy challenges? Evidence from Macedonia, Serbia and Bosnia and Herzegovina. Land Use Policy 59: 71-83.

Tan, Y., J. Jiang and H. Chen. 2005. The effects of different means to disseminate information on agricultural policies and the acceptance by the farmers. Issues in Agricultural Economy 9: 64-67.

Toader, M. and G.V. Roman. 2015. Family farming - examples for rural communities development. Agriculture and Agricultural Science Procedia 6: 89-94.

Turvey, C.G. and X. Xiong. 2017. Financial inclusion, financial education, and e-commerce in rural China. Agribusiness 33(2): 279-285.

Tzouramani, I., A. Liontakis, A. Sintori and G. Alexopoulos. 2014. Assessing organic cherry farmers' strategies under different policy options. Modern Economy 5(4): 313-323.

Vercammen, J. 2007. Farm bankruptcy risk as a link between direct payments and agricultural investment. European Review of Agricultural Economics 34(4): 479-500.

Van de Gucht, T., W. Saeys, A. Van Nuffel, L. Pluym, K. Piccart, L. Lauwers, J. Vangeyte and S. Van Weyenberg. 2017. Farmers' preferences for automatic lameness-detection systems in dairy cattle. Journal of Dairy Science 100(7): 5746-5757.

Vliet, J.A.V., A.G.T. Schut, P. Reidsma, K. Descheemaeker, M. Slingerland, G.W.J. Van de Ven, and K.E. Giller. 2015. De-mystifying family farming: features, diversity and trends across the globe. Global Food Security 5: 11-18.

Wang, D. and S. Tian. 2015. A comparative research on the model of vocational agricultural education for new generation of famers under the background of balanced development of urban and rural regions. Research in Educational Development 9: 70-77.

Wang, O. and J. Yang. 2014. The impact of agricultural subsidization to farmer's households' grain production in China. Chinese Rural Economy 5: 20-28.

Wen, C.H. and S.C. Lai. 2011. Latent class models of international air carrier choice. Transportation Research Part E: Logistics and Transportation Review 46(2): 211-221. 
Wei, Y.F. 2009. Rural land transfer model based on characteristic agricultural industrialization in China - an investigation on the practice of rural land transfer in Fuchuan Guangxi. Rural Economy 8: 35-38.

Wiggins, S., J. Kirsten and L. Llambi. 2010. The future of small farms. World Development 38(10): 1341-1348.

Xie, M. and Q. Zhao. 2016. The evaluation of the effectiveness of the benefit agriculture policy and the strategic thinking. Rural Economy 7: 22-28.

$\mathrm{Xu}, \mathrm{H} .2014$. Research on the family farm cultivation of new agricultural management entities - based on the investigation of Hubei province. Academic Forum 1: 67-71.

Yang, D.T. and M.Y. An. 2002. Human capital, entrepreneurship, and farm household earnings. Journal of Development Economics 68(1): 65-88.

Ye, M.H. and G.Z. Tuo. 2015. Factor input, climate change and food production - based on the dual function model. Journal of Agrotechnical Economics 11: 4-13.

Yin, S., Y. Gao and L. Wu. 2017. Constructing the social security system of food safety with Chinese characteristics: theory and practice. Chinese People's Publishing House, Beijing, China.

Yu, Z. and H. Chen. 2016. Farm, farm system and the development of China's family farm. Journal of Jiangxi University of Finance and Economics 1: 90-96.

Zhang, D. and W. Pan. 2015. Credit provision of family farm and its governance path. Rural Economy 3: 59-63.

Zhao, W., H. Wang and J. Liu. 2015. The development of family farm in China: dilemma and countermeasures. Economic Review 4: 37-41.

Zhu, H., Z. Tian and J. Lou. 2016. An analysis on the prospects and threshold of operation scale of family farm in China. China Agricultural University Journal of Social Sciences 33(6): 65-73.

Zhu, Q., P. Hu and H. Xu. 2014. Discussion about family farm: advantage, requirement and scale. Issues in Agricultural Economy 7: 11-17. 